Pedagogía y Saberes n. ${ }^{\circ} 55$ Universidad Pedagógica Nacional

Facultad de Educación. 2021. pp.121-135

\title{
La desigual distribución de saberes para el trabajo en escuelas secundarias
}

The Unequal Distribution of Knowledge for Work in

Secondary Schools A distribuição desigual de conhecimento para o trabalho nas escolas secundárias

Mauro Guzmán* Eduardo Langer**

Guzmán, M. y Langer, E. (2021). La desigual distribución de saberes para el trabajo en escuelas secundarias. Pedagogía y Saberes, (55). https://doi.org/10.17227/pys.num55-11862

* Profesor en Ciencias de la Educación; becario doctoral Conicet; doctorando en Ciencias de la Educación (UNLP); docente e investigador en Universidad Nacional de la Patagonia Austral. Correo: viktorm_g@hotmail.com

** Doctor en Educación (UBA); magíster en Ciencias Sociales (Flacso), licenciado en Ciencias de la Educación (UBA); investigador adjunto Conicet; director de las carreras de Educación unsam; profesor de Sociología de la Educación en Universidad Nacional de la Patagonia Austral y Universidad Nacional de San Martin. Correo: langereduardo@gmail.com 


\section{Resumen}

El siguiente artículo de investigación indaga sobre la distribución desigual de saberes para el trabajo en tiempos de capitalismo flexible considerando los sentidos de los estudiantes sobre la formación en escuelas secundarias en distintos emplazamientos urbanos. Se presentan resultados de tesis doctoral en curso, referidos al trabajo con encuestas de carácter muestral, cuyo criterio de selección fue el de necesidades básicas insatisfechas (NBI) del emplazamiento de las instituciones escolares. Aquí se focaliza en los sentidos de estudiantes que asisten a escuelas públicas en zonas de mayores niveles de pobreza. Los resultados del artículo remiten a distribuciones desiguales de saberes para el sector productivo, a través de redes de escolarización no segmentadas que expresan relativas correspondencias con las condiciones de pobreza en que se emplazan las escuelas.

\section{Palabras clave}

formación para el trabajo; saberes; distribución desigual; escuela secundaria; estudiantes

\section{Abstract}

The article explores the unequal distribution of knowledge for work in times of flexible capitalism. It focuses on the sense students assign to training in secondary schools in different urban locations. The results of the doctoral thesis in progress referred to work with sample surveys, whose selection criteria were based on the unmet basic needs (NBI in Spanish) of school institutions. Here, the interest is on the students who attend schools in areas with higher levels of poverty. The results of the article refer to unequal distributions of knowledge for the productive sector through non-segmented schooling networks that express relative correspondences with the conditions of poverty in which the schools are located.

\section{Keywords}

training for work; knowledge; uneven distribution; high schools; student

\section{Resumo}

0 artigo de pesquisa indaga sobre a distribuição desigual do conhecimento para o trabalho em tempos de capitalismo flexível, considerando os sentidos dos alunos sobre a formação no Ensino Médio em diferentes localidades urbanas. Apresentamos os resultados de uma tese de doutorado, em andamento, referentes ao trabalho com pesquisas de amostra, cujo critério de seleção foi o de Necessidades Básicas Insatisfeitas (NBI) de instituições escolares. Aqui o centro de atenção está nos sentidos dos alunos que frequentam escolas públicas em áreas com níveis mais elevados de pobreza. Os resultados do artigo referem-se a distribuições desiguais de conhecimento para o setor produtivo, por meio de redes de ensino não segmentadas que expressam correspondência relativa com as condições de pobreza onde as escolas estão localizadas.

\section{Palavras-chave}




\section{Introducción}

Los procesos de formación para el trabajo de los jóvenes se producen, entre otros lugares, en las escuelas secundarias que tienen entre sus objetivos transmitir saberes a la población para la participación en formas más sofisticadas de la vida social, económica y política. La mirada está puesta en cómo estos procesos ocurren en la localidad de Caleta Olivia, cuya matriz productiva y mercado laboral se configura, directa o indirectamente, en relación con la extracción de petróleo. En este marco presentamos resultados de tesis doctoral en curso ${ }^{1}$, a partir de lo cual nos preguntamos por la distribución de saberes para el trabajo desde la formación de nivel secundario.

La discusión actual sobre la formación se produce, siguiendo a Deleuze (1989), en aquello en lo que nos estamos convirtiendo, más que con lo que somos. Es decir, hay que pensar y preguntarse por los lugares de la escuela en los términos de la formación permanente y el control continuo en un momento en el que nada se termina nunca ${ }^{2}$, en un contexto en el que las instituciones son lugares discutidos permanentemente (Deleuze, 2006). Así, cabe preguntarse y tratar de comprender los efectos de las dinámicas de formación en cuanto a la producción en el capitalismo flexible (Harvey, 2000; Castel, 1997; Sennett, 2000) y a las lógicas de gobierno de las conductas en las sociedades de empresa (Foucault, 2007).

Esos interrogantes cobran relevancia a la luz de las transformaciones del contenido y las formas que adopta la formación que se acerca cada vez más a los rasgos no cognitivos de la personalidad a los que hacían referencia Bowles y Gintis $(1985)^{3}$ hace casi cuatro décadas. Está claro que, en la actualidad, la formación está cada vez más asociada a las formas de ser y hacer en términos de competencias. Sin duda,

1 Los resultados de investigación corresponden al plan de tesis doctoral Conicet "Formación para el trabajo en el espacio urbano fragmentado: un estudio en escuelas secundarias de las localidades del Golfo San Jorge", la cual se desarrolla en el marco del Doctorado en Ciencias de la Educación de la Universidad Nacional de La Plata, y cuyas actividades de investigación se inscriben en el Área Sociopedagógica de la Universidad Nacional de la Patagonia Austral-Unidad Académica Caleta Olivia.

2 A diferencia de lo que existe en las sociedades disciplinarias, donde se pasa de manera lineal y progresiva de la escuela al ejército y del ejército a la fábrica, Deleuze (2006) muestra que nunca se termina nada en las sociedades de control. Se pasa de la escuela a la empresa y de la empresa se vuelve a la escuela.

3 Para estos autores, la formación de los sectores obreros se centraba en la enseñanza de actitudes ligadas con los requeriemientos del trabajo obrero: cumplir horario, respetar la autoridad, responder consignas, etc. hoy esas formas se producen según la distribución social de los saberes en el espacio urbano, a las diferenciaciones asociadas a las orientaciones de esos saberes y/o a aquellos que se enseñan en vínculo con el mundo del trabajo. Por ello, aquí la hipótesis a desarrollar es que las lógicas y dinámicas flexibles que se configuran en los ámbitos global y local producen características particulares en la formación de los sujetos, en general, y para el mundo del trabajo, en particular, lo cual profundiza las desigualdades sociales y educativas, y se materializa en las características del espacio urbano (Prevot-Shapira, 2001; Veiga, 2009). Ello cobra particular interés en una región como la del Golfo San Jorge ${ }^{4}$, cuya estructura productiva está asociada al petróleo y que hoy presenta la complejidad de los derrumbes y de las tensiones del mundo globalizado. Dichos derrumbes y tensiones se expresaron con profundidad en los efectos de la privatización de Yacimientos Petrolíferos Fiscales (YPF) ${ }^{5}$ a finales de siglo XX (Márquez, 1995; Salvia, 1997; Ruiz y Muñoz, 2008). Aunque coyunturalmente hubo periodos de mejoramiento y crisis en los últimos años, estructuralmente esos efectos persisten en las luchas que se desarrollan para mejorar las condiciones laborales o evitar el desempleo (Figari y Palermo, 2010; Schweitzer, 2012; Márquez, 2017).

Para ello, en este artículo se trabajan algunos de los resultados a través de una propuesta metodológica de base múltiple y a partir de una combinación de registros cualitativos y cuantitativos de información. La indagación sobre la distribución de los saberes para el trabajo en tiempos de flexibilidad se realiza desde tres tipos de datos que se georreferencian en el espacio urbano: a) datos escolares sobre las orientaciones que se ofrecen en las escuelas secundarias de Caleta Olivia ${ }^{6}$; b) datos de encuestas a estudiantes respecto a los saberes que la escuela está enseñando para su futuro laboral; c) datos sociodemográficos a

4 Golfo San Jorge es el nombre de la cuenca hidrocarburífera conformada por localidades que crecieron al calor de la extracción de petróleo, luego de su descubrimiento a principios del siglo xx en la ciudad de Comodoro Rivadavia, y que actualmente está compuesto por las localidades de: Comodoro Rivadavia, Rada Tilly, Caleta Olivia, Cañadón Seco, Pico Truncado y Las Heras. Comodoro Rivadavia y Caleta Olivia son las localidades con mayor densidad poblacional y superficie urbana de la región.

5 Es una empresa del Estado argentino que desde la década de 1930 y hasta mediados de los años 1990 fue la única empresa encargada de la producción petrolera de Santa Cruz.

6 Hemos trabajado con datos oficiales del Consejo Provincial de Educación de Santa Cruz: http://educacionsantacruz.gov.ar/. 
partir de índices de necesidades básicas insatisfechas $(\mathrm{NBI})^{7}$ que caracterizan al radio censal en que se ubican las escuelas ${ }^{8}$.

Cada uno de los radios censales está compuesto por cierto porcentaje de hogares con NBI. En función de ello, el conjunto de radios censales de la localidad se ha agrupado en cinco quintiles cuyos polos van desde NBI bajas, es decir donde hay menos cantidad de hogares con pobreza, a NBI altas o con mayor cantidad de hogares en situación de pobreza. Estos datos fueron georreferenciados mediante sistemas de información geográfica (SIG), que permitieron adoptar una perspectiva urbana de la desigualdad social y de la educación secundaria en Caleta Olivia.

La muestra con la que se trabajó para la realización de la encuesta fue la selección de una escuela para cada uno de los cinco quintiles de NBI. La selección de la escuela de cada quintil se hizo en función del comportamiento de sus indicadores educativos hacia el extremo negativo. Dentro de cada institución se eligieron al azar un curso del primer año de la secundaria, un curso del primer año del ciclo orientado (tercer año de la secundaria) y un curso del último año del ciclo orientado (quinto año de bachilleratos y sexto año de escuelas técnicas). Este último criterio buscó abarcar la mirada de los estudiantes de todo el nivel, considerando los momentos claves relacionados con el inicio del nivel secundario en general, el inicio del ciclo orientado y la perspectiva de quienes han transitado por todo el nivel y están a punto de finalizar la secundaria y salir hacia el mercado de trabajo. El instrumento de encuesta estuvo compuesto por distintas dimensiones de análisis, las cuales refirieron a las vivencias de los estudiantes sobre su escolaridad y perspectivas a futuro, como las laborales asociadas a su escolaridad. Su implementación se realizó durante 2018, en cinco escuelas públicas de nivel secundario de la localidad de Caleta Olivia, e implicó un alcance de 412 estudiantes.

El análisis de la información relevada se realizó a partir de la utilización del programa informático Spss que permite sistematizar el volumen de información cualitativa y cuantitativa de la totalidad de las encuestas, sin mecanizar el proceso de análisis, y que incluye

7 Dicho indicador es un método directo para identificar carencias críticas en una población y caracterizar la pobreza, que usualmente utiliza indicadores directamente relacionados con cuatro áreas de necesidades básicas de las personas (vivienda, servicios sanitarios, educación básica e ingreso mínimo), información que se obtiene de los censos de población y vivienda (Feres y Mancero, 2001).

8 Se trabajó con los últimos datos disponibles del Indec, que corresponden al censo del año 2010 . desde operaciones sencillas -como distribución de frecuencias o medidas descriptivas- hasta otras más complejas como análisis de conjunto respecto a diferentes variables, dimensiones e indicadores producto de preguntas abiertas y cerradas de la encuesta. Por otra parte, se utilizó el método de comparación constante (Glaser y Strauss, 1967) para la generación y sugerencia plausible de categorías, propiedades e hipótesis sobre el problema que requiere saturación de los datos.

En un primer apartado se presenta, de forma breve, el marco contextual y teórico desde donde se piensa y producen los resultados en torno a las correspondencias entre los cambios en el sector productivo que tiende a la flexibilidad y los requisitos de saberes para el trabajo que realizan las escuelas. En un segundo momento, se describen las formas en que los estudiantes se hacen de los saberes, cuáles creen que son su utilidad en el mercado de trabajo y, en todo caso, qué disputas expresan ante esas correspondencias de las flexibilidades entre el trabajo y la escuela en un mundo que no tiene lugar para todos. Finalmente, presentamos algunas reflexiones finales en torno a los resultados expresados.

\section{Correspondencias entre los cambios en el sector productivo y los requisitos de saberes para el trabajo desde la escolaridad}

Desde fines de siglo $\mathrm{xx}$, las mutaciones del régimen de acumulación del capitalismo presentan modos de producción y distribución de las riquezas a partir de lógicas flexibles donde la precariedad y la desocupación ganan terreno. En el caso específico que nos ocupa, la actividad principal de Caleta Olivia es la petrolera. Aún lo sigue siendo a pesar de los vaivenes y las crisis que vienen sosteniéndose en el sector. Aquí, los trabajadores petroleros han perdido gran parte de las luchas mayormente ganadas por los sindicatos del sector en las décadas de 1950 y 1960, en las que los modos de producción taylor-fordista se caracterizaban por la división de tareas de dirección, concepción, control y ejecución. Hasta los años 1970, las tareas y los saberes de los obreros se asociaban a actividades de repetición y, por tanto, la formación debía estar dirigida al trabajo manual (Harvey, 2000; Sennett, 2009). Tenían aseguradas jornadas laborales de ocho horas y su actividad estaba arraigada. Hoy, sin duda, mucho de todo eso está cambiando. Se extienden las jornadas de trabajo, hay sobrecarga con menos protecciones sociales y las condiciones de precariedad de los trabajadores se van acentuando (Schweitzer, 2012; Márquez, 
2017). Esto ha profundizado ciertas dinámicas preexistentes asociadas a periodos fuera de su contexto social y comunitario y, por tanto, producen un desarraigo muy grande en sus vidas, así como un sentimiento de individualidad muy fuerte (Acconcia y Álvarez, 2008; Márquez, 2008; Grimson y Baeza, 2016).

En el marco de las lógicas productivas anteriores, hace cuatro o cinco décadas los estudios reproductivistas (Baudelot y Establet, 1975; Bowles y Gintis 1985) anunciaban las responsabilidades del sistema escolar en la legitimación de las desigualdades sociales. Se anunciaba la distribución de los estudiantes, según las distintas posiciones del aparato productivo, en dos "redes de escolarización completamente distintas, por las clases sociales a las que están masivamente destinadas, por los puestos de la división social del trabajo y por el tipo de formación que imparten" (Baudelot y Establet, 1975, p. 21). A la vez, Bowles y Gintis (1985) proponían la noción del principio de correspondencia para caracterizar la organización de la escuela y la del mundo del trabajo, en atención, principalmente, a cómo la educación del obrero industrial ocurría a través de los rasgos no cognitivos de la personalidad. Estos autores mostraron cómo las relaciones sociales eran aquello que la instrucción escolar de los circuitos obreros propiciaba. De esta forma, la formación de actitudes era lo que tenía peso en la asignación de los sujetos a las distintas posiciones dentro de la estructura de clase. Pero ese tipo de formación y esas dos redes de escolarización han vivido un proceso de ruptura al compás de la crisis del capitalismo industrial.

Esa crisis involucra el derrumbe de la sociedad salarial así como de cualquier imagen del empleo estable (Castel, 2012; Harvey, 2020). Además, el modelo flexible se basa en la producción just in time que implica poner el acento en "resolución de problemas, en las respuestas rápidas y a menudo altamente especializadas, y en la adaptación de las capacitaciones a propósitos específicos" (Harvey, 2000 , p. 178). En ello ha sido clave la incorporación de la microelectrónica a los procesos productivos, las cuales han modificado las características del trabajo, principalmente el reemplazo de las tareas más simples y repetitivas que pueden ser realizadas por el complejo tecnológico (Coriat, 2011). La intensificación y profundización de la extracción de recursos naturales en la región del Golfo San Jorge, a partir de la proliferación de la participación de actores transnacionales en los últimos años, implica la materialización de estas lógicas (Schweitzer, 2012). La informatización y robotización de los procesos productivos está avanzando en una reconfiguración de todo el circuito petrolero, siguiendo a Márquez
(2017), "con demanda de trabajadores de cada vez mayor calificación y competencia, y la reducción de los planteles en el sector de trabajos repetitivos y rutinarios" (p. 21).

La tendencia global y local, desde ya hace un par de décadas, es reducir el número de trabajadores estables y apelar cada vez más a una fuerza de trabajo que pueda reclutarse y despedirse rápidamente y sin costo cuando los negocios empeoran (Harvey, 2020). Así se produce lo que en su momento Castel (1997) denominó la inempleabilidad de los calificados y la población de supernumerarios. Esto ha estado presente en los efectos de la privatización de la empresa YPF a fines del siglo XX (Salvia, 1997; Ruiz y Muñoz, 2008), y que hoy se profundiza aún más en nuestros barrios de Caleta Olivia con dos o tres generaciones en las familias de desocupados estructurales. Ello debido, además, por las situaciones constantes de huelgas en demanda de mejores puestos laborales en el sector formal de la economía, tanto en el sector petrolero como en la administración pública, y que ha llegado a colocar a Caleta Olivia como la capital nacional del piquete (Márquez, 2017). En este sentido, las dinámicas sociolaborales de la región configuran en las localidades del Golfo San Jorge, sociedades compuestas por

sectores vinculados de alguna manera a la actividad extractiva, con ingresos más altos, otros empleados en el sector público, con ingresos muchos menores y sufriendo el impacto de subas de precios de vivienda y bienes de consumo básico y, finalmente, otros que no encuentran inserción y protagonizan lógicas de protestas por trabajo o son víctimas del clientelismo a cambio de subsidios o bolsas de comida. (Schweitzer, 2012, pp. 47-48)

Por tanto, el auge y la consolidación del capitalismo flexible y la precarización del trabajo han generado, en la ciudad, mutaciones en la vida social, urbana y educativa.

En este marco, las disposiciones que exigen las actividades laborales requieren de un trabajador que, cada vez más, disponga de saberes ligados a la planificación, control, ajuste y mantenimiento. Estudios en la región han mostrado cómo la incorporación de avances tecnológicos en las actividades extractivas desde fines de los años 1990 viene generando cambios en las demandas de disposiciones laborales del operario petrolero, siguiendo a Aranciaga, a través de "una nueva división del trabajo a partir del paradigma informacional de creación de valor, de conectarse con otros trabajadores distantes en el espacio y por último de toma de decisiones en tiempo real" (2004, p. 95). Es decir, se ha generado un desplazamiento de la imagen del obrero petrolero definido exclusivamente 
por actividades manuales y repetidas (Acconcia y Álvarez, 2008), hacia otras identificaciones en términos de polivalencia o tareas múltiples que implican mayores actividades de abstracción (Aranciaga, 2004; Riquelme, 2014). Podríamos decir que ello refiere a un proceso general de inmaterilización del modo de producción capitalista, siguiendo a Lazzarato y Negri (2001), debido a la actividad abstracta que remite a la subjetividad del trabajador. El obrero es llamado a decidir, "siendo su principal actividad la de gestionar los distintos tipos de información, optando por la más conveniente" (Giordano y Cató, 2012, p. 15).

Por otra parte, si en el auge de la industrialización del siglo XVIII hubo "una conexión íntima entre el desarrollo del capitalismo y el proceso de urbanización" (Harvey, 2014, p. 22), así como el problema del gobierno de los hombres era en parte el de la escolarización de la población (Foucault, 2006), en el presente ya no se trata de las totalizaciones en torno a procesos de homogeneización. Es decir, la vida urbana toda se ha visto atravesada por cambios en los usos y prácticas en y del espacio urbano. Nos referimos a la conformación de las grandes metrópolis y la distribución de las poblaciones en condición de pobreza (Prevot-Shapira, 2001; Veiga, 2009; Wacquant, 2001), desde las cuales no podemos centrarnos, como hace algunas décadas, en el análisis centro/periferia para caracterizar sus dinámicas. Ello porque hay estrategias defensivas de los sectores empobrecidos que ocupan territorios céntricos $y$, a la vez, aparición de formas residenciales que se emplazan en las periferias, tal como viene sucediendo en Caleta Olivia. Al respecto, Pérez (2013) menciona que, a partir de las dinámicas sociales producto de la situación de crisis del petróleo en la región, el paisaje urbano de Caleta Olivia comienza a dibujarse por

\begin{abstract}
clases medias o medias altas que buscan y demandan al mercado inmobiliario espacios "seguros" y de "calidad" para el "buen vivir"; por el otro, [...] espacios devaluados o de "riesgo" surgidos a partir de las demandas de obreros desocupados o subocupados, "piqueteros", "madres solteras" o nuevos migrantes sin redes de contención serán, entre otros, los elementos emergentes del polarizado paisaje urbano. (Pérez, 2013, p. 26)
\end{abstract}

Así, siguiendo la figura 1, la distribución de la pobreza por NBI en el espacio urbano de la localidad en estudio muestra que la mayoría de los emplazamientos más pobres se ubican en las zonas periféricas de la ciudad. Los sectores que se emplazan en estos barrios se caracterizan por ser mayormente poblaciones migrantes del norte del país y países limítrofes en búsqueda de mejores condiciones de vida (Baeza, 2015), como también sectores empo- brecidos que han llevado a cabo estrategias de asentamientos informales en esos barrios (Moraga, 2014; Bachiller, 2016).
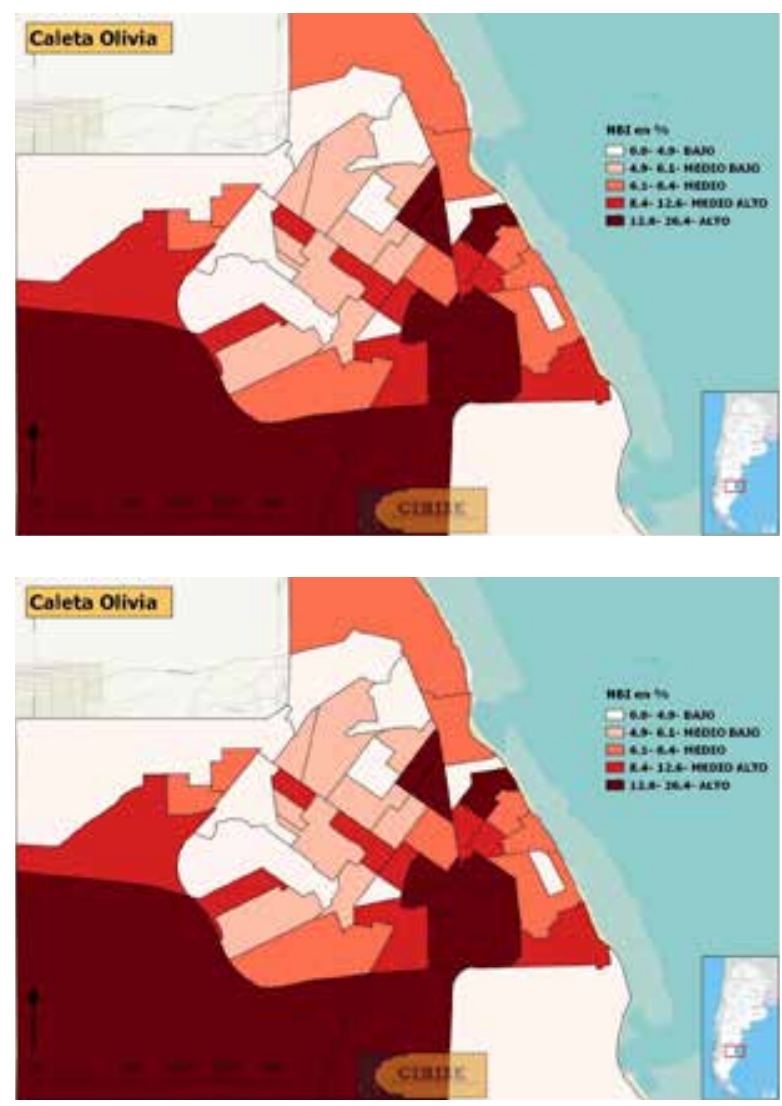

Figura 1. Distribución de radios censales con hogares con NBı. Caleta Olivia, 2010

Fuente: elaboración propia en base a Centro de Intercambio y Reservorio de Información Social y Educativa, e censo nacional Indec (2010).

También es posible visualizar algunos radios censales con NBI altas en las zonas céntricas, como también emplazamientos con NBI bajas en los límites urbanos. Es decir, la distribución de la pobreza urbana en Caleta Olivia se caracteriza por cierta persistencia de lógicas centro/periferia, que coexisten con dinámicas menos lineales y más fragmentadas de expansión de la mancha urbana. De allí que la escolaridad hay que describirla y pensarla en torno a la multiplicidad de esas formas que adopta lo urbano hoy. Entre los espacios a evitar (Osborne y Rose, 1999), los pozos urbanos infernales donde "solo los parias de la sociedad tolerarían vivir" (Waquant, 2001, p.179) y en donde las reconfiguraciones territoriales profundizan los desencuentros entre sectores sociales (Veiga, 2009). 
Es en esas comunidades donde se asientan acciones de gobierno en que el Estado no interviene más que para garantizar/gerenciar que esos lazos comunitarios sucedan. No en la sociedad como un todo, sino en una moral que refuerza los lazos individuales y comunitarios (Grinberg, 2008; Noguera-Ramírez y Marín-Díaz, 2011). Por tanto, hay capacidades, denominadas competencias por los sectores laborales, que antes los sujetos no debían disponer para desarrollarse en el mercado productivo. Nos referimos a aquellas de autogestión para hacerse de los recursos con los cuales sortear obstáculos y, a partir de allí responsabilizarse por los resultados (Grinberg, 2008; Castro-Gómez, 2010). Al respecto, Noguera-Ramírez y Marín-Díaz señalan que hoy el "aprendizaje con énfasis en la gubernamentalidad neoliberal” (2012, pp. 16-17) se centra en las competencias que desarrollen capacidades y habilidades para aprender a conocer, a hacer, a vivir, a ser y disposición para gerenciar las propias posibilidades con cierto capital humano que se puede y debe acrecentar.

Por tanto, en cuanto al ámbito laboral, la relación entre los saberes y el trabajo hace ya varios años que se viene estructurando no en base a la noción de calificaciones, asociadas a tareas específicas, saberes duraderos y que serían propios del modo de producción taylor-fordista (Spinoza, 2006; Brunet y Zavaro, 2014). Más bien, es la noción de competencias desarrollada anteriormente la que, en tiempos de capitalismo flexible y gubernamentalidad neoliberal, cobra peso para pensar los saberes y disposiciones claves tanto en las inserciones laborales (Jacinto y Millenaar, 2012), como en las trayectorias en el mercado formal de trabajo en general (Montes, 2018; Loureiro y Lopes, 2019), e incluso para sobrellevar el desempleo como situación duradera (Grinberg, 2008). Los procesos de reestructuración en la organización del trabajo en las actividades extractivas de la región del Golfo San Jorge estuvieron marcadas, y aun lo están, por estas tensiones entre calificaciones y competencias (Álvarez, 2008; Ruiz y Muñoz, 2008). Si hasta entrados los años 1980 era posible una clara identificación de las posiciones en la organización del trabajo asociadas a las calificaciones de los empleados (Álvarez, 2008), con los procesos de privatización esto comienza a desdibujarse. Los extrabajadores del petróleo, siguiendo a Ruiz y Muñoz, entendieron agónicamente que sus saberes del trabajo solo eran aplicables al mundo laboral del que provenían y que ya no está, así "la gestión sostenida en la intensificación del trabajo ligada a la polivalencia y la multifuncionalidad, trajo aparejada la necesidad de capacitación permanente y la formación en el puesto de trabajo" (2008, p. 113).
Ello conlleva una demanda al sistema educativo regional, tal como revisaremos a continuación, en términos elevación de los niveles de calificación, sin la garantía ni la posibilidad de que los sujetos obtengan esos lugares una vez finalizada su educación, a la vez que también la especificidad en el problema de los saberes que las orientaciones van ofreciendo en las diferentes escolaridades que se van configurando en Caleta Olivia.

Por tanto, ante este escenario global, regional y local nos preguntamos ¿cuál es el lugar de las correspondencias entre formación escolar y mundo del trabajo hoy, y particularmente en la localidad de Caleta Olivia?; ¿qué características asumen esas redes de escolarización en el escenario del capitalismo flexible?; ¿cómo se relacionan esas caracterizaciones con la profundización de la desigualdad y precariedad en la región? Aquí daremos respuestas a estos interrogantes a partir de las miradas que tienen los estudiantes sobre sus procesos de escolaridad y del mercado de trabajo que les (y no que los) espera. Estas perspectivas se analizarán en función de la distribución urbana de la oferta escolar y de los saberes ante las condiciones socioeconómicas del espacio urbano.

\section{Los saberes para el mundo del trabajo desde los sentidos estudiantiles}

En los últimos años, se están produciendo redes de escolarización heterogéneas y diversas del nivel secundario en Caleta Olivia como parte de las localidades más pobladas del Golfo de San Jorge y que parecieran manifestar una misma lógica de conformación. Las diferenciaciones se realizan sobre la relación entre la distribución de los índices escolares asociados con las condiciones de pobreza en que se ubican las escuelas y las lecturas que aquí se proponen tienen que ver con la posibilidad de un estallido o ruptura de aquellas redes de escolarización duales que los teóricos de la correspondencia (Bowles y Gintis, 1985; Baudelot y Establet, 1975) presentaban como circuitos diferenciados de formación para el trabajo y que los caracterizaban como circuitos paralelos en función del rendimiento escolar.

En la actualidad, estas redes expresan una tendencia a diferenciarse entre sí, justamente a partir de los saberes que se ofrecen en el tipo de formación de las distintas orientaciones escolares de las instituciones. En otros trabajos, hemos descrito las formas en que la distribución de las orientaciones se vincula a la posición geoespacial de la escuela y, también, en relación con una dimensión socioeconómica (Guzmán, 
Langer y Grinberg, 2020). Por ejemplo, en Caleta Olivia las orientaciones en Artes Visuales y la de Educación Física solo se ofrecen en escuelas ubicadas en las zonas con mayor cantidad de hogares con NBI, mientras que la orientación en Economía y Administración solo se ofrece en las escuelas ubicadas en los sectores con menores niveles de pobreza. Teniendo en cuenta que las formaciones orientadas se dirigen hacia determinados campos de saber y campos laborales, al menos desde la planificación política de las escuelas, esto implica pensarlas en su articulación con el mercado laboral de la región. Es decir, hay racionalidades políticas (Foucault, 2006; Castro-Gómez, 2010) en el mundo educativo que ponen en juego determinadas tecnologías de gobierno (Foucault, 2006), con la finalidad de desarrollar un campo del conocimiento. De allí que esas maneras en que se distribuyen las orientaciones en Caleta Olivia permiten preguntarnos por las orientaciones de aquellos estudiantes de las escuelas en mayores condiciones de pobreza, que no serían las más demandadas por el mercado de trabajo de la región. Dicho de otra manera, aquí el interrogante es si esas planificaciones o racionalidades están o no ligadas a lo productivo dado que, tal como lo anunció Foucault (2007), las sociedades de empresa configuran una gubernamentalidad que no se centra ante todo en la producción, ya que no se necesitan muchos brazos con la condición de que todos trabajen. El sector que se viene describiendo puede ser un ejemplo significativo de esa reducción.

Ahora bien, entendemos que el devenir de lo escolar implica también procesos de disputas de sentidos (Deleuze, 1989; Scott, 2000; Langer, 2017) por parte de los actores que dan vida a aquello que se presenta como la dimensión estructural-formal del currículum escolar (De Alba, 2006). En ese marco, interesa indagar a continuación aquello que los estudiantes de escuelas secundarias de Caleta Olivia expresan a propósito de los saberes que les están enseñando desde la escuela y para el mundo del trabajo. Para ello, consideramos un primer elemento analítico desde las perspectivas de los estudiantes a partir del lugar que ocupa la orientación de la escuela a la que asisten y la relación con el mundo del trabajo en relación con los niveles de pobreza de emplazamiento escolar, tal como se detalla en la tabla 1.

Tabla 1. Razones por las que estudiantes asisten a la escuela en la que se encuentran, según NBI de las escuelas. Caleta Olivia, 2018. En \%. N=412

\begin{tabular}{|l|c|c|c|c|c|}
\multicolumn{1}{c|}{ RAZONES } & \multicolumn{3}{c|}{ NBI } \\
\cline { 2 - 6 } \multicolumn{1}{c|}{} & Bajas & Medio bajas & Medio & Medio altas & Altas \\
\hline Le interesa la orientación & 39,1 & 72,6 & 18,2 & 14,6 & 18,2 \\
\hline Se relaciona con lo que quiere trabajar & 32,1 & 63,5 & 25,0 & 17,1 & 18,2 \\
\hline Es prestigiosa/recomendada/reconocida & 39,1 & 55,7 & 13,6 & 17,1 & 11,4 \\
\hline Hay un buen ambiente social y cultural & 35,9 & 31,5 & 22,7 & 26,8 & 27,3 \\
\hline \multicolumn{1}{|c|}{ Totales } & 100 & 100 & 100 & 100 & 100 \\
\hline
\end{tabular}

Fuente: elaboración propia. Datos del Centro de Intercambio y Reservorio de Información Social y Educativo (Cirise), Área Sociopedagógica, UNPA-UACo.

Los estudiantes que asisten a la escuela por motivos asociados a la orientación o al trabajo son mayormente los de escuelas ubicadas en zonas con menores NBI (NBI bajas: 39,1\% y 32,1\%, respectivamente; NBI medio bajas: $72,6 \%$ y $63,5 \%$, respectivamente). En cambio, si bien el porcentaje de los estudiantes que asisten a escuelas con mayores necesidades (NBI medio altas y altas) es menor, son muchos en proporción quienes relacionan su asistencia a la escuela al interés por la orientación y la relación con el trabajo (NBI medio altas: $14,6 \%$ y 17,1\%, respectivamente; NBI altas: $18,2 \%$ en ambas variables). La mayoría de los estudiantes que asisten a las escuelas ubicadas en emplazamientos con altos niveles de pobreza no han elegido su escuela principalmente por la orientación que ofrece o por su relación con el mundo del trabajo. Se podría pensar que ellos y ellas en un primer momento no la eligen, pero "cuando ya están allí sí lo hacen por aquello que la escuela es y por aquello que allí viven" (Langer, 2013, p. 379). Al revés de lo que sucede con sus pares que concurren a escuelas con NBI bajas ya que en mayor medida asisten porque hay buen ambiente social y cultural, porque es prestigiosa, recomendada o reconocida. 
Es decir, no solo hay un vínculo entre distribución de orientaciones y emplazamiento urbano de la escuela, sino que esta relación también tiene correspondencias con los sentidos que los estudiantes expresan sobre la orientación que ofrece su escuela y cómo se relaciona con el futuro laboral. De esta manera, en aquellas escuelas ubicadas en zonas con altos niveles de pobreza, las orientaciones que se ofrecen únicamente allí, tal como ya dijimos, artes visuales y educación física, tienen una especie de menor jerarquía como campo de conocimiento o en su relación con el sistema productivo de la región basado en la actividad petrolera, en comparación con la que se ofrece solo en escuelas emplazadas con niveles de pobreza bajo que es la de Economía y Administración. Ello se produce porque durante los años de mayor crecimiento y dinamismo en la economía de la región luego del periodo privatizador de fines del siglo xx (entre 2003 y 2007), se han generado empleos formales en sectores no asociados a perfiles dentro de las Artes Visuales y/o Educación Física: construcción, actividades extractivas, comercio, sector servicios en actividades inmobiliarias, transporte y comunicaciones (Rojo y Rotondo, 2009). Sumado a lo anterior, cabe remarcar que, como estrategia de tercerización de las grandes empresas y también de las estrategias de los extrabajadores del petróleo, se ha producido la creación de numerosas cooperativas que luego devinieron en pequeñas empresas que ofrecen servicios a la actividad petrolera (Prado y Robledo, 2010; Márquez, 2017). Estas, sumadas a pequeñas y medianas empresas regionales basadas en actividades comerciales y de servicio en general, representaban el $90 \%$ del sector productivo empresarial en la región para el 2009 (Prado y Robledo, 2010).

\section{Sentidos sobre la enseñanza de la escuela para el mundo del trabajo}

Si profundizamos el análisis en torno a aquello que los estudiantes expresan sobre la enseñanza de los saberes para el mundo del trabajo en la escuela, observamos en la tabla 2 muchas semejanzas y diferencias entre instituciones con distintas condiciones de emplazamiento.

Tabla 2. Saberes que enseña la escuela para el mundo del trabajo, según NBI de las escuelas. Caleta Olivia, 2018. En \%. $N=412$

\begin{tabular}{|c|c|c|c|c|c|}
\hline \multirow{2}{*}{ Saberes } & \multicolumn{5}{|c|}{ NBI } \\
\hline & Bajas & Medio bajas & Medio & Medio altas & Altas \\
\hline Disciplina & 57,8 & 44,2 & 81,8 & 19,5 & 27,2 \\
\hline Estudiar/Información & 15,6 & 18,2 & 18,1 & 2,4 & 4,5 \\
\hline Trabajar en grupo & 17,8 & 15 & 25 & 4,8 & 9 \\
\hline No responde & 15,6 & 14,1 & 13,6 & 48,7 & 36,3 \\
\hline Saberes básicos y generales & 35,9 & 4,1 & 22,7 & 26,8 & 34 \\
\hline Uso de las Tic/informática & 4,6 & 10,5 & 2,2 & 0 & 0 \\
\hline Otros idiomas (Inglés) & 4,6 & 1,8 & 2,2 & 0 & 0 \\
\hline Esforzarse/Sacrificio & 3,1 & 5 & 4,5 & 0 & 2,2 \\
\hline Relacionado a la orientación y el título & 18,7 & 10,5 & 9 & 4,8 & 22,7 \\
\hline Hacer por mi cuenta & 4,6 & 1,3 & 0 & 0 & 2,2 \\
\hline Saberes técnicos/Oficios & 3,1 & 56,8 & 11,3 & 2,4 & 11,3 \\
\hline Buena persona/Buen ciudadano & 4,6 & 6,3 & 9 & 7,3 & 4,5 \\
\hline Competencias comunicativas & 12,5 & 9,5 & 11,3 & 2,4 & 6,8 \\
\hline Pensar/Solucionar problemas & 0 & 3,1 & 4,5 & 0 & 4,5 \\
\hline No lo sé & 15,6 & 5 & 0 & 2,4 & 4,5 \\
\hline Todo me sirve & 4,6 & 0 & 4,5 & 7,3 & 4,5 \\
\hline En nada & 1,6 & 2,7 & 6,8 & 4,8 & 0 \\
\hline
\end{tabular}

Fuente: elaboración propia. Datos del Centro de Intercambio y Reservorio de Información Social y Educativo (Cirise), Área Sociopedagógica, UNPA-UACo. 
En cuanto al primer grupo, al de las semejanzas, encontramos aquellos sentidos asociados a la transmisión de saberes básicos y generales que realiza la escuela, a la orientación y el título, y al ser buena persona o buen ciudadano. No es menor, desde el punto de vista que se viene sosteniendo, que se produzcan estas miradas en relación directa a los contenidos o a los rasgos cognoscitivos que transmiten las escuelas sin distinción por el emplazamiento o los niveles de pobreza, dado el perfil productivo que se viene configurando en esta ciudad.

En cambio, al abordar las diferencias entre las distintas instituciones, vemos que la disciplina, estudiar, trabajar en grupo, el uso de las TIC, idiomas y la transmisión de saberes de oficios son los sentidos que expresan diferencialmente los estudiantes con relación al lugar de emplazamiento. La disciplina -en términos de puntualidad, respeto de las consignas, la responsabilidad, etc.- implica una gran importancia para estudiantes que asisten a establecimientos con niveles bajos de pobreza (NBI bajas: $57,8 \%$, NBI medio bajas: $44,2 \%$ y NBI medio: $88,1 \%$ ). Los porcentajes casi duplican aquello que expresan estudiantes que concurren a escuelas con NBI medio altas y altas (19,5 \% y $27,2 \%$, respectivamente).

Como decíamos al principio del artículo, la enseñanza de los saberes no cognoscitivos que se asocian al sostenimiento de un trabajo, como cumplir con las tareas o llegar a horario, teniendo en cuenta los sentidos de los estudiantes, está íntimamente relacionado al emplazamiento escolar. Quienes menos sienten que le están enseñando disciplina para el trabajo desde la escuela son estudiantes que asisten a instituciones en zonas con alto nivel de pobreza. Es la formación de los supernumerarios o los que no van a tener lugar en un sector cada vez más competitivo e injusto.

La tabla 2 también expresa algunas particularidades con respecto a los saberes útiles para el mundo del trabajo, como obtener mayor información o saber trabajar en grupo, que se suceden mayormente entre los estudiantes que asisten a escuelas ubicadas en NBI bajas (15,6\% y 17,8 \%, respectivamente), NBI medio bajas (18,2\%y $15 \%$,respectivamente) y NBI medio $(18,1 \%$ y $25 \%$, respectivamente). En contraposición, los porcentajes disminuyen al considerar las miradas de quienes van a escuelas ubicadas en NBI medio altas $(2,4 \%$ y $4,8 \%$, respectivamente) y NBI altas $(4,5 \%$ y $9 \%$, respectivamente). Estos saberes están relacionados con las características del proceso productivo del posfordismo que requieren un trabajador polivalente, un tipo de trabajo que implica el grupo y las exigencias de actualización permanente, siguiendo las dinámicas del mercado de trabajo, tal como fueron las descripciones de los pri- meros apartados. Es decir, al igual que con los sentidos sobre la disciplina, los estudiantes que asisten a escuelas en zonas con altos niveles de pobreza expresan ciertas formas de persistencia de la desigualdad respecto al acercamiento y a la enseñanza de estos saberes, en comparación con aquellos que asisten a escuelas con NBI más bajas. Por ello, observamos en otros estudios (Langer y Esses, 2019) que los estudiantes son quienes demandan tener más y mejor educación, que quieren tener clases y que sus docentes les enseñen.

Lo mismo sucede con los saberes asociados a las tecnologías, la informática y los idiomas. No solo que los mayores porcentajes están entre quienes asisten a escuelas ubicadas en NBI bajas $(4,6 \%$ en ambos casos), NBI medio bajas $(10,5 \%$ y $1,8 \%$, respectivamente) y NBI medio (2,2 \% en ambos casos), sino que nadie de los que asisten a escuelas ubicadas en NBI medio altas y NBI altas percibe que su escuela le esté enseñando algo respecto a esos saberes para el trabajo. Enmarcamos estas resignaciones juveniles en torno a que la informática, la tecnología, la microelectrónica y la globalidad de los idiomas son dimensiones claves sobre las que se asientan las mutaciones de los procesos productivos y, sobre todo, forman parte neurálgica de las actividades de los trabajos más estables (Coriat, 2011; Harvey, 2020). Ello sucede en el mercado productivo local en el que, como mencionamos, los avances en el complejo tecnológico forman parte de la organización del trabajo y saberes de trabajadores que las empresas petroleras, y también las prestadoras de servicios (Prado y Robledo, 2010), buscan para intensificar el retorno de ganancia y ser competitivas (Aranciaga, 2004; Márquez, 2017). Para profundizar la analítica que estos sentidos expresan en cuanto a ciertos pesimismos en relación con lo que les enseña la escuela para el mundo del trabajo, son los estudiantes de escuelas ubicadas en zonas con NBI altas y NBI medio altas quienes mayormente no responden esta pregunta. De hecho, la categoría "no responde" de la tabla es la que mayor porcentaje presenta en las escuelas ubicadas en esos NBI (NBI medio altas: 48,7 \%; NBI altas: $36,3 \%$ ).

Entre estas tensiones, entre los señalamientos y las pugnas por las desigualdades y las resignaciones o pesimismos, enmarcamos los saberes para el mundo del trabajo que los estudiantes perciben sobre aquello que la escuela les está enseñando y, como dijimos, guardan una estrecha relación con las características del emplazamiento urbano de las instituciones. Los resultados nos permiten indicar que entre esas contradicciones persisten las condiciones de desigualdad para la formación para el trabajo en el marco del capitalismo flexible. Esta lectura se acentúa 
al observar en la tabla 3, en la que se expresan tres grados de consideración (de "muy bien" a "regular") que los estudiantes manifiestan con respecto a las formas como la escuela los prepara para distintas cosas, pero que no necesariamente tienen que ver con el mundo del trabajo.

\section{Las distintas consideraciones sobre los saberes que se transmite en la escuela}

Tabla 3. Grados de consideración de los saberes que transmite la escuela, según NBI de las escuelas. Caleta Olivia, 2018. En \%. N = 412

\begin{tabular}{|c|c|c|c|c|c|c|c|c|c|c|c|c|c|c|c|}
\hline & \multicolumn{15}{|c|}{ NBI } \\
\hline & \multicolumn{3}{|c|}{ Bajas } & \multicolumn{3}{|c|}{ Medio bajas } & \multicolumn{3}{|c|}{ Medio } & \multicolumn{3}{|c|}{ Medio altas } & \multicolumn{3}{|c|}{ Altas } \\
\hline & $\begin{array}{l}\frac{0}{0} \\
\frac{\partial}{3} \\
\frac{2}{\Sigma}\end{array}$ & ฮี & 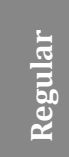 & $\frac{\overline{0}}{0}$ & ฮี & 志 & 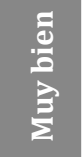 & 馬 & 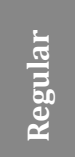 & $\frac{\sigma}{0}$ & 可 & 离 & $\frac{\tilde{\omega}}{\partial}$ & 需 & 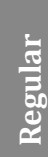 \\
\hline $\begin{array}{l}\text { Trabajar en } \\
\text { equipo }\end{array}$ & 33,7 & 42 & 16,9 & 35,2 & 47 & 14,2 & 18,2 & 46 & 29,5 & 17,1 & 39 & 9,8 & 36 & 32 & 16 \\
\hline $\begin{array}{l}\text { Coordinar un } \\
\text { grupo de trabajo }\end{array}$ & 26,5 & 34 & 26,5 & 27,4 & 41 & 25,6 & 15,9 & 43 & 27,3 & 9,8 & 37 & 22 & 25 & 23 & 18 \\
\hline $\begin{array}{l}\text { Utilizar las } \\
\text { tecnologías }\end{array}$ & 25,3 & 42 & 14,5 & 33,8 & 37 & 21,9 & 13,6 & 30 & 45,5 & 17,1 & 29 & 17,1 & 18 & 30 & 27 \\
\hline $\begin{array}{l}\text { Usar técnicas } \\
\text { de estudio }\end{array}$ & 34,9 & 37 & 16,9 & 30,1 & 43 & 22,4 & 18,2 & 57 & 18,2 & 22 & 34 & 14,6 & 23 & 27 & 18 \\
\hline $\begin{array}{l}\text { El esfuerzo para } \\
\text { superarme }\end{array}$ & 47 & 28 & 13,3 & 35,2 & 31 & 19,2 & 31,8 & 48 & 13,6 & 22 & 29 & 14,6 & 48 & 25 & 2,3 \\
\hline $\begin{array}{l}\text { Conocer la cul- } \\
\text { tura del trabajo }\end{array}$ & 39,8 & 29 & 16,9 & 26 & 39 & 25,6 & 18,2 & 48 & 22,7 & 17,1 & 46 & 7,3 & 27 & 23 & 18 \\
\hline
\end{tabular}

Fuente: elaboración propia. Datos del Centro de Intercambio y Reservorio de Información Social y Educativo (Cirise), Área Sociopedagógica, UNPA-UACo.

Trabajar en equipo, coordinarlo, utilizar tecnologías y conocer o valorar las formas culturales que adquiere el trabajo hoy, son sin duda parte de las dimensiones centrales que constituyen las competencias que el proceso productivo requiere en la actualidad. Diversos estudios en la región (Rojo y Rotondo, 2009; Acconcia et ál., 2010; Romero y Galaretto, 2013) muestran cómo esos saberes mencionados configuran los perfiles y atributos buscados por sectores públicos y privados para la contratación de fuerza de trabajo, configurando "mecanismos diferenciales de selección, contratación, capacitación de personal, etc., que responden a características vinculadas a modelos de organización empresarial" (Acconcia et ál., 2010, p. 27). En correspondencia, la escuela se propone enseñar estos saberes y valores.
Pero pareciera que, de nuevo, ello sucede diferencialmente con desiguales grados de consideración y de realización en las instituciones de distintos emplazamientos urbanos de acuerdo con las miradas de los estudiantes.

Con estos sentidos, se observa que quienes mayormente consideran que la escuela los prepara para el trabajo en equipo son, en primer lugar, estudiantes que asisten a escuelas ubicadas en NBI altas (36\%); en segundo lugar, los de NBI medio bajas $(35,2 \%)$ y en tercer lugar los de NBI bajas (33,7\%). Algo semejante ocurre en relación con la preparación para coordinar un grupo de trabajo, que si bien los porcentajes más altos están en NBI medio bajas $(27,4 \%)$ y NBI bajas $(26,5 \%)$; luego son los estudiantes que van a escuelas con NBI altas con un porcentaje similar (25\%), 
quienes piensan que la escuela los forma para ello. Como ya mencionamos, hoy poseer estos saberes es una condición necesaria, pero no suficiente, tanto para la inserción laboral como para pensar en las características de esos puestos laborales. La distribución de la enseñanza de estos tipos de saberes permite la indagación y tener algunas pistas sobre la formación de los sectores más estables del mercado laboral y de sus zonas periféricas.

En este punto, y en línea con lo anterior, cabe remarcar que se produce una diferencia con aquello que expresaba la tabla 2. Allí, los saberes asociados al trabajo en grupo tenían un peso marcadamente inferior entre los estudiantes de escuelas en NBI altas, en comparación con lo que expresaban quienes asisten a instituciones en NBI bajas y medio bajas. Es decir, pareciera que el trabajo en grupo es parte de esos saberes que los estudiantes que concurren a escuelas con mayores niveles de pobreza perciben como aprendizajes que suceden en la escuela, pero que no específicamente tienen relación con el mundo del trabajo.

De forma similar ocurre con la preparación y utilización de las tecnologías y de técnicas de estudio. Los estudiantes manifestaron que los preparan muy bien en esos saberes en escuelas con NBI altas $(18 \%$ y $23 \%$, respectivamente) y medio alto $(17,1 \%$ y $14,6 \%$, respectivamente), son menos en proporción que aquellos que concurren a instituciones con NBI bajas $(25,3 \%$ y $34,9 \%)$ y NBI medio bajas $(33,8 \%$ y $30,1 \%$, respectivamente). Sin embargo, cuando comparamos con lo observado en la tabla 2 , hay una gran diferencia en los datos. Las técnicas de estudio son consideradas positivamente para el $22 \%$ de estudiantes de escuelas en NBI medio altas y altas. Ahora bien, a la hora de pensar este saber en relación con el mundo del trabajo, volviendo a la tabla 2 , solo el $2,4 \%$ y el $4,5 \%$ de estudiantes de NBI medio altas y altas, respectivamente, lo mencionaron. En cuanto al uso de las tecnologías, ningún estudiante de NBI altas o medio altas manifestó que fuera un saber que la escuela les esté enseñando para el mundo del trabajo tal como se expresa en la tabla 2, mientras que cerca del $50 \%$ de estudiantes para ambos NBI expresan que la escuela los prepara muy bien o bien en el uso de las tecnologías en la tabla 3. Es decir, hay grandes diferencias entre lo que perciben que sucede dentro de la escuela y lo que tiene relación con el mundo del trabajo. Esta disociación, sostenemos, es propia de las lógicas de formación que se configuran racionalmente hacia la actualidad, una formación desligada del mundo productivo que está siendo.

Por último, se observan las consideraciones sobre el lugar que ocupa la escuela en la formación para la cultura del trabajo y para el esfuerzo o la superación, donde hay casi una indistinción en las miradas de estudiantes que concurren a escuelas con distintos NBI. A partir de las respuestas positivas en conjunto, los porcentajes en todos los casos van de 68,7 \% en instituciones de NBI bajas a $63,4 \%$ en aquellas que tienen NBI medio altas. En el caso de la formación para el esfuerzo y la superación, los porcentajes oscilan entre $69 \%$ en NBI bajas a $73 \%$ en NBI altas. Es decir, no hay una correlación entre estos saberes y valores con el emplazamiento urbano de la escuela. 0 , más bien, ello sucede en un marco en el que los valores del capitalismo flexible y de las teorías meritocráticas y de las competencias se han instalado en el todo social, más allá de las condiciones de vida y las persistencias institucionales por solucionar problemas profundos de desigualdad. Esos valores dan cuenta, como describíamos con anterioridad, el sostenimiento del individualismo y la competencia en las tareas del sector productivo predominante en Caleta Olivia, produciendo desarraigo, individualismo y competencia entre los sujetos trabajadores de la ciudad.

Los estudiantes de escuelas de distintas zonas, incluso las de altos índices de pobreza, perciben que saberes como el trabajo en grupo, el uso de tecnologías, el aprender a aprender o bien los valores de la cultura del trabajo y del esfuerzo para poder superarse, son enseñados allí. Incluso hay una gran mayoría que manifiesta que la escuela los prepara bien o muy bien en ellos. Sin embargo, estos mismos estudiantes son los que no vinculan esos saberes a los sentidos de utilidad para el futuro laboral, ya que no se suceden con la misma fuerza ni peso (o incluso algunos ni siquiera han aparecido) como saberes que se están enseñando para el mundo del trabajo.

No nos llama la atención esta brecha encontrada en y desde sus propias miradas. Los discursos gubernamentales, las lógicas productivas en términos de flexibilidad y competencias, así como los discursos meritocráticos y del esfuerzo calan profundamente en las subjetividades de los trabajadores de la ciudad, de sus familias y, por supuesto, de los jóvenes en formación. Claro que allí hay disputas y contrasentidos que habrá que seguir explorando en los tiempos presentes en que los estudiantes rescatan que hay escuelas que efectivamente transmiten saberes, y los tiempos futuros en los que ellos viven incertidumbres, miedos y frustraciones frente a un mundo del trabajo flexible que es cruel, sanguinario y que no tiene lugar para todos.

\section{Consideraciones finales}

En este trabajo se buscó analizar las formas en que las dinámicas flexibles producen características particulares en la formación de los sujetos en general y 
para el mundo del trabajo en particular, atendiendo a los sentidos que los estudiantes dan de los saberes y sus utilidades para el futuro laboral. Entre esas miradas detectamos diferenciaciones estrechas en relación con los niveles de pobreza del emplazamiento escolar que a continuación mencionamos.

La asociación entre escuela y trabajo se exploró, en un primer momento, a partir de los saberes para el trabajo que piensan los estudiantes que les enseñan, y en un segundo momento, en relación con grados de consideración sobre la formación más allá o desligado de lo laboral.

En un primer momento, observamos asociaciones fuertes con estudiantes que asisten a escuelas ubicadas en zonas con necesidades básicas insatisfechas bajas y asociaciones más débiles en los jóvenes que concurren a escuelas en emplazamientos con necesidades altas. En estas diferencias también suceden gradaciones jerárquicas en los campos de conocimiento o en su relación con el sistema productivo entre estudiantes de diferentes emplazamientos urbanos. Allí se expresan sentidos distintos aunque no excluyentes que mencionamos a continuación. Un sentido que se expresa en una mayoría de estudiantes que asisten a las escuelas ubicadas en emplazamientos con altos niveles de pobreza es que no han elegido la escuela a la que asisten por su relación con el mundo del trabajo. Algo que, en cambio, sí sucede en mayor medida entre las razones de los estudiantes que van a escuelas ubicadas en zonas con bajos niveles de pobreza. Otro sentido se expresa en torno a la enseñanza de aquellos saberes sobre el sostenimiento de un trabajo, tales como cumplir con las tareas o llegar a horario, y que se relacionan estrechamente con las características de emplazamiento escolar. Quienes perciben con menos fuerza qué se les están enseñando desde la escuela son estudiantes que asisten a instituciones ubicadas en zonas con alto nivel de pobreza. Es decir, son esos jóvenes que se encuentran en condición de desigualdad respecto al aprendizaje de estos saberes en comparación con los estudiantes de escuelas con menores necesidades.

En cuanto al segundo momento, en la perspectiva de estudiantes de escuelas ubicadas en zonas con las NBI más altas, tienen menos peso aquellos saberes que adquieren relevancia para el mundo del trabajo en el capitalismo flexible. Ello, quizás, porque saben quiénes son, dónde viven, qué futuro les depara. Tal como observamos en otras investigaciones que hemos realizado (Langer, 2013), los estudiantes que transitan su escolaridad en contextos de pobreza urbana se saben los supernumerarios (Castel, 1997), que el mercado laboral no los incluye y que, a pesar de todo, siguen estando en la escuela. Por ello, otro de los resultados centrales de este escrito es respecto a sus percepciones sobre cómo la escuela los prepara en cultura del trabajo y esfuerzo para superarse. En estas dimensiones las correlaciones fuertes que encontrábamos con el emplazamiento urbano de la escuela a la que asisten se debilitan. Ello porque la cultura del trabajo y el esfuerzo o la superación son dimensiones transversales que son parte de aquello que transmite la escuela hoy, pero fundamentalmente porque son parte de los discursos conservadores y expulsivos que circulan en el todo social cotidianamente.

En estos dos momentos, rescatamos pesimismos, tensiones, contradicciones, disputas, luchas, miedos, frustraciones y esperanzas ante sus formaciones y los saberes que circulan en el sistema educativo y que les serán de utilidad para sus futuros próximos. Entre estos sentidos, las redes de escolarización en la ciudad expresan una distribución de saberes y perspectivas sobre el mundo del trabajo que coloca a quienes transitan la escolaridad secundaria en condiciones de pobreza no necesariamente como potenciales obreros o como ejército de reserva que compite por entrar a trabajar en esas condiciones, sino como potenciales supernumerarios o inútiles para el mundo (Castel, 1997). Pero lejos de considerar ello como parte de destinos sociales y escolares inmodificables o inexorables, lo escolar no solo está marcado por estas lógicas que se imponen como acción de gobierno escolar, sino principalmente por las dinámicas de luchas y disputas que los sujetos ejercen en esa búsqueda e insistencia por ocupar lugares en un sector productivo en crisis.

\section{Referencias}

Acconcia, M. y Álvarez, M. (2008). El ser ypefiano. Una identidad al abrigo de la Empresa (o una identidad territorializada). En J. D. Ruiz (coord.), Petróleo y región austral: saberes del trabajo y educación técnica. Reconfiguraciones y nuevas subjetividades (pp. 65-90). Editorial Dunken.

Acconcia, M., Álvarez, M., Michniuk, N. y Villanueva, M. (2010). Pensar la escuela de cara al siglo XXI. Debates no saldados en el marco de las reformas educativas: una mirada desde la provincia de Santa Cruz. Congreso Iberoamericano de Educación: Metas 2021. Buenos Aires.

Álvarez, M. (2008). YPF en la Región Austral: saberes, trabajo y perfiles laborales. En J. D. Ruiz (coord.), Petróleo y región austral: saberes del trabajo y educación técnica. Reconfiguraciones y nuevas subjetividades (pp. 119137). Buenos Aires: Editorial Dunken.

Aranciaga, I. (2004). Tecnología y sociedad en el petróleo de la Patagonia argentina. [Tesis de maestría]. UNPA, Caleta Olivia, Argentina. 
Bachiller, S. (2016). Petróleo, dificultades de acceso al suelo urbano y toma de tierras: un problema recurrente en la historia comodorense. Revista Identidades, (6), 71-87.

Baeza, B. (2015). Identificaciones y territorialización de migrantes quechuas de Bolivia en Caleta Olivia, Santa Cruz, Argentina. Revista de Geografía Norte Grande, (62), 109-126.

Baudelot, C. y Establet, R. (1975). La escuela capitalista. Siglo XXI Editores.

Bowles, S. y Gintis, H. (1985). La instrucción escolar en la América capitalista. Siglo XxI Editores.

Brunet, I. y Zavaro, B. (2014) Competitividad, competencias y fin del ciclo fordista. Revista Internacional de Sociología de la Educación, 3(1), 1-25.

Castel, R. (1997). La metamorfosis de la cuestión social. Una crónica del salariado. Paidós.

Castel, R. (2012). El ascenso de las incertidumbres: trabajo, protecciones, estatuto del individuo. Fondo de Cultura Económica.

Castro-Gómez, S. (2010). Historia de la gubernamentalidad. Razón de Estado, liberalismo y neoliberalismo en Michel Foucault. Siglo del Hombre Editores.

Coriat, B. (2011). El taller y el robot. Ensayos sobre el fordismo y la producción en masa en la era de la electrónica. Siglo XXI Editores.

De Alba, A. (2006). Currículum: crisis, mito y perspectiva. Miño y Dávila.

Deleuze, G. (1989). Lógica del sentido. Paidós.

Deleuze, G. (2006). Post-scriptum sobre las sociedades de control. Polis, Revista Latinoamericana, 13. Recuperado de http://journals.openedition.org/polis/5509

Feres, J. C. y Mancero, X. (2001). El método de las necesidades básicas insatisfechas (NBI) y sus aplicaciones en América Latina. Cepal.

Figari, C. y Palermo, H. (2010). Producción y reproducción de sentidos en un enclave petrolero. La privatización de YPF en Comodoro Rivadavia. Revista Trabajo, 4(6),146-165.

Foucault, M. (2006). Seguridad, territorio y población. Fondo de Cultura Económica.

Foucault, M. (2007). Nacimiento de la biopolítica. Fondo de Cultura Económica.

Giordano, P. y Cató, J. (2012). Diez tesis sobre el trabajo inmaterial. Revista GPT: Gestión de las Personas y Tecnología, (14), 17-31.

Glaser, B. y Strauss, A. (1967). The discovery of grounded theory. Aldine Publishing Company.
Grimson, A. y Baeza, B. (2016). Desacoples entre nivel de ingresos y jerarquías simbólicas en Comodoro Rivadavia. Acerca de las legitimidades de la desigualdad social. Revista Identidades, 6(10), 01-21.

Grinberg, S. (2008). Educación y poder en el siglo XXI. Gubernamentalidad y pedagogía en las sociedades de gerenciamiento. Miño y Dávila.

Guzmán, M., Langer, E. y Grinberg, S. (2020). Educación, trabajo y redes de escolarización en el capitalismo flexible: un estudio en localidades del Golfo San Jorge. Revista Trabajo y Sociedad, XXI(35), 603-623.

Harvey, D. (2000). La condición de la posmodernidad. Investigación sobre los orígenes del cambio cultural. Amorrortu

Harvey, D. (2014). Ciudades rebeldes. Ediciones Akal.

Harvey, D. (2020). Razones para ser anticapitalistas. Clacso.

Instituto Nacional de Estadísticas y Censos de la República Argentina (Indec) (2010). Censo Nacional de Población, Hogares y Viviendas 2010.

Jacinto, C. y Millenaar, V. (2012). Los nuevos saberes para la inserción laboral. Formación para el trabajo con jóvenes vulnerables en Argentina. Revista Mexicana de Investigación Educativa, 17(52), 141-166.

Langer, E. (2013) Prácticas de resistencia de estudiantes y regulación de las conductas en escuelas secundarias en contextos de pobreza urbana del Partido de San Martín. [Tesis de Doctorado en Ciencias de la Educación]. Universidad Nacional de Buenos Aires.

Langer, E. (2017). Escuela, pobreza y resistencia. Defensas y luchas cotidianas de estudiantes. Del Gato Gris.

Langer, E. y Esses, J. (2019). La salida es por arriba. Una historia de juventud, pobreza y educación. Grupo Editor Universitario.

Lazzarato, M. y Negri, A. (2001). Trabajo inmaterial. Formas de vida y producción de subjetividad. DPyA Editora.

Loureiro, C. y Lopes, M. (2019). Aprendizagem: o imperativo de uma nova ordem econômica e social para o desenvolvimento. Pedagogía y Saberes, (51), 89-102.

Márquez, D. (1995). Crisis socio-laboral y políticas de empleo: Algunas reflexiones para el análisis del caso comodorense. En D. Márquez y M. Palma-Godoy, Distinguir y comprender: aportes para pensar la sociedad y la cultura en Patagonia (pp. 169-194). Edición Proyecciones Patagónicas.

Márquez, D. (2008). La constitución de una "gran familia": trabajadores e identidades sociolaborales. Ponencia presentada en III Jornadas de Historia de la Patagonia. Bariloche. 
Márquez, D. (2017). Del petróleo estatal al petróleo privado: continuidades y rupturas en el mundo sociolaboral de los trabajadores petroleros de la cuenca del Golfo San Jorge durante las últimas tres décadas. Ponencia presentada en XVI Jornadas Interescuelas, Universidad de Mar del Plata.

Montes, C. (2018). Perfil de egreso y empleo en el contexto del avance tecnológico. Pedagogía y Saberes, (48), 83-96.

Moraga, J. (2014). La conformación de asentamientos informales en Caleta Olivia: un estudio particular a partir del análisis de las teorías de la exclusión social. ICT UNPA, (79), 37-58.

Noguera-Ramírez, C. y Marín-Díaz, D. (2011). Educar es gobernar. En R. A. Cortés Salcedo y D. L. Marín Díaz (comp.), Gubernamentalidad y educación. Discusiones contemporáneas. Investigación e Innovación.

Noguera-Ramírez, C. y Marín-Díaz, D. (2012). Educar es gobernar: la educación como arte de gobierno. Cuadernos de Pesquisa, (42), 14-29.

Osborne, T. y Rose, N. (1999). Governing cities: notes on the spatialisation of virtue. Environment and Planning D: Society and Space, (17), 737-760.

Pérez, A. (2013). Políticas de escolarización: un acercamiento al análisis de la oferta escolar en clave de territorio. En S. Grinberg (coord.), La escuela not dead. Dispositivos pedagógicos, territorios y desigualdad (pp. 21-38). Ediciones Universidad Nacional de la Patagonia Austral.

Prado, M. y Robledo, M. (2010). La actividad hidrocarburífera en la cuenca del Golfo San Jorge: el papel del empresariado regional. Revista de Estudios Regionales y Mercado de Trabajo, (6), 117-193.

Prevot-Schapira, M. F. (2001). Fragmentación espacial y social: conceptos y realidades. Revista Perfilaes Latinoamericanos, (19), 33-56.

Riquelme, M. (2014). Trabajadores petroleros de la empresa YPF una postura diferente frente a la nación. En Actas Pre Alas Patagonia 2014, Estado, sujetos y desigualdad en América Latina. Un debate en torno de la desigualdad (S/D). El Calafate. https://prealas2014. unpa.edu.ar/sites/prealas2014.unpa.edu.ar/files/ ckeditor/46/Acta\%20de\%20Res\%c3\%bamenes\%20 Mesa\%20\%205.pdf

Rojo, S. y Rotondo, S. (2009). Perfil de especialización del empleo registrado en el Golfo San Jorge: un diagnóstico desde la perspectiva del desarrollo local. En Actas de $9^{\circ}$ Congreso Nacional de Estudios del Trabajo (pp. 1-20). Buenos Aires. https://www.aset.org.ar/congresos/9/ Ponencias/p1_Rojo.pdf

Romero, J. y Galaretto, M. (2013). Nuevas tecnologías, nuevos escenarios educativos y laborales en la zona norte de Santa Cruz. UNPA Edita

Ruiz, J. D. y Muñoz, N. (2008). La privatización de YPF y su repercusión en las subjetividades. En J. D. Ruiz (coord.), Petróleo y región austral: saberes del trabajo y educación técnica. Reconfiguraciones y nuevas subjetividades (pp. 91-118). Buenos Aires: Editorial Dunken.

Salvia, A. (1997). Crisis y reestructuración de complejos mineros: estudios de dos sistemas regionales patagónicos. En A. Salvia y M. Panaia (comp.), La Patagonia privatizada (pp. 36-48). Colección CEA-CBC.

Schweitzer, A. (2012). Petróleo y territorios en la Provincia de Santa Cruz. Aproximaciones al estudio del espacio del Golfo de San Jorge. En M. Álvarez, N. Michniuk y M. Villanueva (coord.), Educación y trabajo, miradas desde lo regional. Territorio y desigualdad de oportunidades (pp. 21-54). Editorial El Colectivo.

Scott, J. (2000). Los dominados y el arte de la resistencia. Ediciones Era.

Sennett, R. (2000). La corrosión del carácter. Las consecuencias personales del trabajo en el nuevo capitalismo. Anagrama.

Sennett, R. (2009). El artesano. Editorial Anagrama.

Veiga, D. (2009). Desigualdades sociales y fragmentación urbana. En H. Poggiese y T. Cohen-Egler (comp.), Otro desarrollo urbano: ciudad incluyente, justicia social y gestión democrática (pp. 51-61). Clacso.

Wacquant, L. (2001). Parias urbanos. Marginalidad en la ciudad a comienzos del milenio. Manantial. 